BRX-TH 478

\title{
Inconsistencies of Massive Charged Gravitating Higher Spins
}

\author{
S. DESER ${ }^{\sharp}$ AND A. WALDRON ${ }^{b}$ \\ \#Physics Department, Brandeis University, \\ Waltham, MA 02454, USA \\ deserebrandeis.edu \\ ${ }^{b}$ Department of Mathematics, University of California, \\ Davis, CA 95616, USA \\ wally@math.ucdavis.edu
}

(February 1, 2008)

\begin{abstract}
We examine the causality and degrees of freedom (DoF) problems encountered by charged, gravitating, massive higher spin fields. For spin $s=3 / 2$, making the metric dynamical yields improved causality bounds. These involve only the mass, the product $e M_{P}$ of the charge and Planck mass and the cosmological constant $\Lambda$. The bounds are themselves related to a gauge invariance of the timelike component of the field equation at the onset of acausality. While propagation is causal in arbitrary E/M backgrounds, the allowed mass ranges of parameters are of Planck order. Generically, interacting spins $s>3 / 2$ are subject to DoF violations as well as to acausality; the former must be overcome before analysis of the latter can even begin. Here we review both difficulties for charged $s=2$ and show that while a $g$-factor of $1 / 2$ solves the DoF problem, acausality persists for any $g$. Separately we establish that no $s=2$ theory - DoF preserving or otherwise can be tree unitary.
\end{abstract}




\section{Introduction}

Localized, massive $s>1$ particles have never been observed, in agreement with a large (if somewhat confusing) higher spin lore that they cannot be made to interact consistently even with gravity or electromagnetism. Here we combine two earlier lines of analysis into a systematic study of higher spins coupled to Einstein-Maxwell fields.

A previous examinations of neutral higher spins propagating in cosmological, as well as $s=3 / 2$ in electromagnetic (E/M), backgrounds reveal the following: (i) Massive higher spins propagate consistently in constant curvature backgrounds for a range of parameters $\left(m^{2}, \Lambda\right)$ centered around the Minkowski line $\left(m^{2}, 0\right)[1$, 2, 3, 4, 5]. (ii) The original unitarity [6] (or equivalently causality [7]) difficulties of massive $s=3 / 2$ persist in pure E/M backgrounds, even including all possible non-minimal couplings [8].

Our first new result is that the onset of the unitarity/causality difficulty for massive $s=3 / 2$ in pure E/M backgrounds can be traced to a novel gauge invariance of the timelike component of the Rarita-Schwinger equation at E/M field strengths tuned to the mass. Although the full system is not invariant, a consequence of this invariance is signal propagation with lightlike characteristics. Beyond this tuned point, i.e., for large enough magnetic field $\vec{B}^{2}>\left(\frac{3 m^{2}}{2 e}\right)^{2}$ (or better, small/large enough mass/charge), the system is neither causal nor unitary. This is an old result but its rederivation in terms of a gauge invariance is edifying. [Higher spin models in constant curvature backgrounds also enjoy unexpected gauge invariances at values of the mass tuned to the background, i.e. to the cosmological constant. The flat space limit $m^{2} \gg \Lambda$ is, of course, always causal, unitary and massive.] Our second $s=3 / 2$ result is that in dynamical Maxwell-Einstein backgrounds, causality can be maintained for any choice of E/M field, for certain values of the mass: It is useful, in this context to first consider the $\left(m^{2}, \Lambda\right)$ phase diagram in Figure 1 for neutral (Majorana) $s=3 / 2$ in a cosmological background [3, 4]. The strictly massless line $m^{2}=-\Lambda / 3$ divides the plane into unitarily allowed and forbidden regions, with the indicated DoF and corresponds to linearized $\mathcal{N}=1$ cosmological SUGRA [9]. The addition of an on-shell Maxwell background shifts the line and we will obtain the new phase diagram depicted in Figure 2 valid for charged (Dirac) systems (the indicated DoF are doubled in going from Majorana to Dirac when counting real components). Hence, causality and unitarity can be maintained for a charged massive $s=3 / 2$ field, but only at the cost of Planck scale masses. 




Figure 1: Phase diagram for neutral, massive $s=3 / 2$ in cosmological backgrounds.

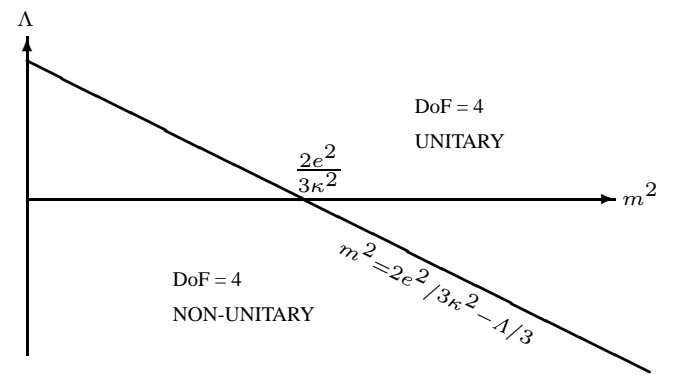

Figure 2: Phase diagram for charged, massive $s=3 / 2$ coupled to cosmological Einstein-Maxwell backgrounds. 


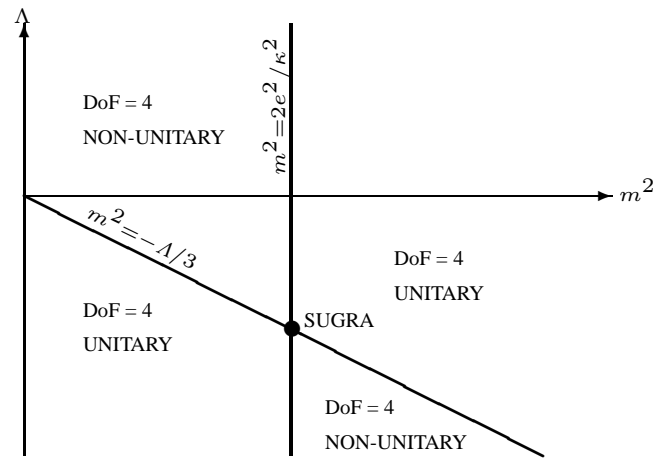

Figure 3: Phase diagram for charged, massive $s=3 / 2$ with SUGRA-inspired non-minimal coupling to cosmological Maxwell-Einstein backgrounds.

The requirement of Planck scale masses for causal propagation is unaffected by further non-minimal couplings as demonstrated by analyzing the characteristic surfaces when a SUGRA-inspired non-minimal magnetic moment coupling is added. However, the structure of the $\left(m^{2}, \Lambda\right)$ phase diagram (see Figure 3) is richer in this case: The point $m^{2}=-3 \Lambda=2 e^{2} / \kappa$ corresponds to the tunings of charge, mass and cosmological constant required for local supersymmetry of charged cosmological gravitini (linearized $\mathcal{N}=2$ SUGRA [[10]). Causality is maintained along the line $m^{2}=2 e^{2} / \kappa^{2}$ for any value of $\Lambda$ [11]. For the region around flat space $\Lambda \approx 0$, causality can only be achieved for Planck mass gravitini, $m^{2} \geq 2 e^{2} / \kappa^{2}$, as mentioned above. Ironically, extremely light, charged gravitini with $m^{2}<-\Lambda / 3$ and $m^{2} \leq 2 e^{2} / \kappa^{2}$ do propagate causally. Observe that the unitarily forbidden region of the neutral $s=3 / 2$ theory [3], 4] is split in two in the presence of SUGRA-inspired E/M interactions. The new unitary region is not useful for effective theories of $s=3 / 2$ excitations since the allowed masses are very small.

It is natural to query whether the rich structure exhibited by the massive $s=$ $3 / 2$ system in Maxwell-Einstein backgrounds is generic to higher spins or specifically inherited from $\mathcal{N}=2$ cosmological SUGRA. The question is interesting from a phenomenological standpoint also, since one would like to develop effective field theoretical methods for relativistic higher spins interacting with E/M backgrounds. Therefore, we also perform an analysis of charged $s=2$ and confirm the suspicion that $s=3 / 2$ is exceptional and can be viewed as a softly broken version of $\mathcal{N}=2$ cosmological SUGRA, rather than a predictor of generic higher 
spin properties.

Higher spins $\geq 2$ actually suffer a much more serious difficulty than acausality à la $s=3 / 2$. Manifestly relativistic descriptions of higher spins demand an ever increasing set of field components as compared to actual physical DoF. Free actions yielding a consistent set of constraints eliminating the unphysical components are known for all spins, massive and massless [12, 13, 14, 15, 16]. However, the consistency of these constraints is not guaranteed in the presence of covariant derivatives associated with gauge interactions. This problem, observed first in the context of massive charged $s=2$ [17], is that constraints may disappear and the number of propagating DoF is discontinuous from that of the underlying free theory - obviously a pathological situation. Although for charged $s=2$ the DoF problem was cured [177] in flat space by introducing a (unique) $g=1 / 2$ magnetic moment coupling, there is no DoF-preserving gravitational interaction in general gravitational backgrounds [18, 19, 20]. The problem is only compounded as the spin increases, requiring consistency of an ever larger set of constraints. It is not even clear whether any DoF preserving coupling to electromagnetic or (not purely cosmological) gravitational backgrounds exists at all for $s \geq 5 / 2$. For $s=2$, this means that there is no analog of the improved $s=3 / 2$ causality bounds which relied on dynamically coupled Maxwell-Einstein backgrounds to use equivalence between Einstein and E/M stress tensors. Furthermore, the pure E/M $g=1 / 2$ model is acausal [21, 22]. For completeness we review the computation of its characteristics.

Finally, we consider the separate problem of tree unitarity for spins $s \geq 2$. Previous authors have suggested that a novel $g=2$ magnetic moment coupling yields improved tree unitarity properties [23]. In fact, not only does this coupling fail to preserve the correct DoF count, but as we will explicitly show, it cannot yield tree unitary Compton scattering amplitudes. Taken as a whole, our results highlight the difficulties associated with an effective description of higher spins by local quantum field theories.

The material is laid out as follows. Section 2 contains our new causality bounds for the charged gravitating massive $s=3 / 2$ system. Minimal, SUGRAinspired non-minimal and generic couplings are treated in Sections 2.1, 2.2 and 2.3, respectively. Spin 2 is dealt with in Section $\beta$, beginning with a review of the DoF results of [17] in Section 3.1. Our streamlined acausality proof for E/M backgrounds may be found in Section 3.2 and a detailed discussion of tree unitarity in terms of the $g$-factor is in Section 3.3. Our conclusions are summarized in Section 4 . 


\section{Charged Gravitating Spin 3/2}

The massive Rarita-Schwinger field $\psi_{\mu}$ is the original instance of acausality in external E/M backgrounds [6, 7]. It is known to maintain the correct $(2 s+1=4)$ DoF count so long as its non-minimal coupling is of the form [24, 8]

$$
\mathcal{L}_{\mathrm{NM}} \sim \bar{\psi}_{\mu} \mathcal{F}^{\mu \nu} \psi_{\nu}
$$

where $\xi_{\mu} \mathcal{F}^{\mu \nu} \xi_{\nu}=0$ for any timelike vector $\xi_{\mu}$. It follows that the timelike component $\xi . \psi$ of $\psi_{\mu}$ is a Lagrange multiplier (imposing a constraint); otherwise terms quadratic in $\xi . \psi$ appear and imply unwanted, propagating DoF. In particular, minimal coupling to gravity and electromagnetism respects the requirement (11).

In this Section we study causal propagation for $s=3 / 2$ in both E/M and gravitational backgrounds. We take gravity and electromagnetism to be dynamical, so that the Maxwell stress tensor is equivalent to the Einstein tensor. [We mostly ignore back-reaction on the $s=3 / 2$ probe field itself, but will also briefly discuss the case where all fields are dynamical.] The main result is that once gravity is dynamical, $s=3 / 2$ causality can be regained at the cost of a Planck scale mass $m$. The responsible mechanism is related to the consistency of the underlying $\mathcal{N}=2$ (broken) SUGRA theory. We also show that the original E/M acausality is caused by a novel gauge invariance of certain field equations.

\subsection{Minimal Coupling}

The massive $s=3 / 2$ Lagrangian minimally coupled to both gravity and electromagnetism is

$$
\mathcal{L}=-\sqrt{-g} \bar{\psi}_{\mu} \gamma^{\mu \nu \rho} \mathcal{D}_{\nu} \psi_{\rho}
$$

where the mass term is included in the extended covariant derivative,

$$
\mathcal{D}_{\mu} \equiv D_{\mu}+\frac{m}{2} \gamma_{\mu}
$$

satisfying $\left[\mathcal{D}_{\mu}, \mathcal{D}_{\nu}\right]=\left[D_{\mu}, D_{\nu}\right]+\frac{m^{2}}{2} \gamma_{\mu \nu}$. The usual covariant derivative on the Rarita-Schwinger field reads

$$
D_{\mu} \psi_{\nu}=\partial_{\mu} \psi_{\nu}-\Gamma_{\mu \nu}^{\rho} \psi_{\rho}+\frac{1}{4} \omega_{\mu m n} \gamma^{m n} \psi_{\nu}+i e A_{\mu} \psi_{\nu}
$$

\footnotetext{
${ }^{1}$ Our metric is "mostly plus" and Dirac matrices are in turn "mostly hermitean", in particular $\gamma^{5}=\gamma^{5 \dagger}=-i \gamma^{0123}=\frac{i}{24 \sqrt{-g}} \gamma_{\mu \nu \rho \sigma} \epsilon^{\mu \nu \rho \sigma}$. We (anti)symmetrize with unit weight.
} 
with commutator

$$
\left[D_{\mu}, D_{\nu}\right] \psi_{\rho}=-R_{\mu \nu \rho}^{\sigma}(g) \psi_{\sigma}+\frac{1}{4} R_{\mu \nu m n}(\omega) \gamma^{m n} \psi_{\rho}+i e F_{\mu \nu} \psi_{\rho}
$$

We generally drop the labels $g$ and $\omega$ with the curvature convention $R_{\mu \nu \rho \sigma} \equiv$ $R_{\mu \nu \rho \sigma}(g)=-e_{\rho}^{a} e_{\sigma}^{b} R_{\mu \nu a b}(\omega)$. The Rarita-Schwinger equation (following from (2)) is

$$
R^{\mu} \equiv \gamma^{\mu \nu \rho} \mathcal{D}_{\nu} \psi_{\rho}=0
$$

We first note that causality is equivalent to the consistency of constraints for all values of the background fields. Along the lines of [3], 4], we can study the problem by searching for gauge invariances: Consider the case of a pure E/M background and search for a gauge invariance

$$
\delta \psi_{\mu}=\mathcal{D}_{\mu} \varepsilon
$$

Varying (6), we find, in terms of the dual $\widetilde{F}^{\mu \nu} \equiv \frac{1}{2} \epsilon^{\mu \nu \alpha \beta} F_{\alpha \beta}$,

$$
\delta R^{\mu}=-\left(e \gamma^{5} \widetilde{F}^{\mu \nu} \gamma_{\nu}+\frac{3 m^{2}}{2} \gamma^{\mu}\right) \varepsilon \equiv \Pi^{\mu} \varepsilon .
$$

It is easy to verify that there is no simultaneous eigenspinor $\varepsilon$ of the operator $\Pi^{\mu}$ with vanishing eigenvalue for each value of the Lorentz index $\mu$ : the massive Rarita-Schwinger equation is not gauge invariant under (7). However, if we just take the timelike component $\mu=0$ and examine the determinant of the matrix multiplying $\varepsilon$ in the variation (8) we find

$$
\operatorname{det} \Pi^{0}=e^{4}\left(\vec{B}^{2}-\left[\frac{3 m^{2}}{2 e}\right]^{2}\right)^{2} .
$$

Hence when

$$
B \equiv|\vec{B}|=\frac{3 m^{2}}{2 e}
$$

and $\varepsilon$ is an eigenspinor of $\Pi^{0}$ with vanishing eigenvalue, the transformation (7) is an invariance of the timelike component of the Rarita-Schwinger equation. Although (7) is not an invariance of the full field equations (or action), this suffices to ruin the consistency of the constraints and permit luminal signal propagation. The bound (10) on the magnetic field is precisely the one discovered in [6, 7] and occurs when the mass is tuned to the external background, just as happened when $m^{2}$ was tuned to the constant curvature gravitational background [3, \#]. 
Next we perform an explicit analysis of the system's characteristics using the method first introduced in this context in [25, 26]. By studying a shock whose first derivative is discontinuous across the wavefront, we may determine the maximum speed of propagation. The leading discontinuities across the characteristic (or wavefront) $\Sigma$ are denoted by square brackets

$$
\left[\partial_{\mu} \psi_{\nu}\right]_{\Sigma} \equiv \xi_{\mu} \Psi_{\nu}
$$

where $\xi_{\mu}$ is a vector normal to the characteristic and $\Psi_{\nu}$ is some non-vanishing vector-spinor defined on the characteristic surface. Propagation is acausal whenever the field equations admit characteristics with timelike $\xi_{\mu}$. Examining the discontinuity in the field equation (6) and its gamma-trace we find

$$
\begin{aligned}
{\left[R_{\mu}-\frac{1}{2} \gamma_{\mu} \gamma \cdot R\right]_{\Sigma} } & =\notin \Psi_{\mu}-\xi_{\mu} \gamma \cdot \Psi=0, \\
{[\gamma \cdot R]_{\Sigma} } & =2(\sharp \gamma \cdot \Psi-\xi \cdot \Psi)=0,
\end{aligned}
$$

which combine to give

$$
\xi^{2} \Psi_{\mu}=\xi_{\mu} \xi . \Psi
$$

Clearly there are two possibilities: The first is vanishing $\xi . \Psi$ and $\xi^{2}$; the maximal speed of propagation is then governed by the light-cone and the model is causal. Alternatively $\xi . \Psi \neq 0$; we proceed by contradiction and assume that $\xi_{\mu}$ is timelike $\left(\xi^{2}=-1\right)$ : To determine when a timelike normal vector to the characteristic is allowed, we consider the secondary (Lagrangian) constraint

$$
\mathcal{D} \cdot R=-\frac{3}{2} m^{2} \gamma \cdot \psi+e \gamma^{5} \gamma \cdot \widetilde{F} \cdot \psi-\frac{1}{2} \gamma \cdot G \cdot \psi
$$

Taking a further derivative and computing the discontinuity we learn

$$
\xi^{\mu}\left[\partial_{\mu} \mathcal{D} \cdot R\right]_{\Sigma}=-\frac{3}{2} m^{2} \gamma \cdot\left[\xi+\frac{1}{3 m^{2}} G \cdot \xi+\frac{2 e}{3 m^{2}} \gamma^{5} \tilde{F} \cdot \xi\right] \xi . \Psi .
$$

The model is acausal if (15) admits a solution for non-zero $\xi . \Psi$. Since for any pair of vectors $\left(v_{\mu}, a_{\mu}\right), \operatorname{det}\left[\gamma^{\mu}\left(v_{\mu}+\gamma^{5} a_{\mu}\right)\right] \equiv(v+a)^{2}(v-a)^{2}$, we are led to study the solubility of

$$
\begin{aligned}
\xi^{2} & +\frac{2}{3 m^{2}} \xi \cdot G \cdot \xi+\frac{1}{\left(3 m^{2}\right)^{2}} \xi \cdot G \cdot G \cdot \xi \\
& \pm\left(\frac{2}{3 m^{2}}\right)^{2} e \xi \cdot G \cdot \widetilde{F} \cdot \xi-\left(\frac{2 e}{3 m^{2}}\right)^{2} \xi \cdot \widetilde{F} \cdot \widetilde{F} \cdot \xi=0
\end{aligned}
$$


Without loss of generality we take $\xi_{\mu}=(1,0,0,0)$ and impose Einstein's equations on the background fields

$$
G^{00}=-\kappa^{2} T^{00}=-\left(\kappa^{2} / 2\right)\left(\vec{E}^{2}+\vec{B}^{2}\right), \quad G^{0 i}=-\kappa^{2} T^{0 i}=-\kappa^{2}(\vec{E} \times \vec{B})^{i} .
$$

The condition (17) then becomes (the \pm term vanishes on-shell)

$$
\begin{gathered}
(1+\mathcal{E}+\mathcal{B})^{2}-4 \mathcal{E B} \sin ^{2} \theta-2 Q \mathcal{B}=0, \\
Q \equiv \frac{4 e^{2}}{3 \kappa^{2} m^{2}}, \quad \mathcal{E} \equiv \frac{\kappa^{2} \vec{E}^{2}}{6 m^{2}}, \quad \mathcal{B} \equiv \frac{\kappa^{2} \vec{B}^{2}}{6 m^{2}}
\end{gathered}
$$

( $\theta$ is the angle between $\vec{E}$ and $\vec{B}$ ). When the gravitational interaction is turned off $(\kappa=0)$ only the terms $1-2 Q \mathcal{B}$ survive and yield the original causality bound [7]] on the magnetic field, $\vec{B}^{2}<\left(\frac{3 m^{2}}{2 e}\right)^{2}$. However for any $\kappa \neq 0$, there are values of $(e, m)$ such that $(19)$ has no solutions for any E/M field strength $F_{\mu \nu}$, and the model is causal. This is the case when the bound/

$$
Q<2 \Longleftrightarrow m^{2}>\frac{2}{3} \frac{e^{2}}{\kappa^{2}}
$$

is satisfied. Since masses of this Planckian order would invalidate our no backreaction assumption, we prefer to state our result as follows: massive $s=3 / 2$ minimally coupled to gravity and electromagnetism cannot propagate causally for mass to charge ratios smaller than the Planck mass. We will discuss the extension to back-reaction at the end of this Section.

The addition of a cosmological constant term shifts the mass term $m^{2} \rightarrow m^{2}+\Lambda / 3$ and modifies the bound to read $m^{2} Q<2\left(m^{2}+\Lambda / 3\right)$ so that

$$
m^{2}>\frac{2 e^{2}}{3 \kappa^{2}}-\frac{\Lambda}{3}
$$

These results yield the $\left(m^{2}, \Lambda\right)$ phase diagram of Figure 2 .

\subsection{SUGRA Causality}

$\mathcal{N}=2$ AdS SUGRA [10] describes a Dirac gravitino coupled (both minimally and non-minimally) to electromagnetism and gravity with cosmological constant.

\footnotetext{
${ }^{2}$ Consider the 1.h.s. of (19) as a quadratic in $\mathcal{B}$ (vanishing $\mathcal{B}$ is always causal) and require that the discriminant be negative, which yields $Q<2+2 \mathcal{E} \cos ^{2} \theta$, whose most stringent bound on $Q$ in 20 is attained for $\vec{E} \perp \vec{B}$.
} 
The apparent mass term for the gravitino has precisely the coefficient required to ensure true masslessness in the presence of a tuned cosmological constant [11]. However, supersymmetry can be softly broken by turning off the cosmological constant without losing causal propagation [11], i.e., keeping the $s=3 / 2$ mass term and detuning the cosmological constant from its supersymmetric value. This implies 4 massive $s=3 / 2 \mathrm{DoF}$ and, in effect, yields a consistent (albeit very heavy) charged massive $s=3 / 2$ model.

One might wonder whether this mechanism can be employed to derive consistent phenomenologically applicable models. Unfortunately the result of [11] also requires a Planck mass gravitino: Although the cosmological constant term was removed, the gravitino mass parameter kept its supersymmetrically tuned value $m=\sqrt{2} e M_{P} \equiv \sqrt{2} e / \kappa$. Here we study the more general case where both the gravitino mass and cosmological constant terms are detuned. The final result is, however, similar to that of [11]: Causality requires the $s=3 / 2$ mass to be either so large or so small that the model cannot be applied to physical situations. In the causal large mass regime, the lower bound on the mass is the supersymmetric value $m>\sqrt{2} e / \kappa$, whilst causal small mass models require an AdS background with an upper mass bound $m<\sqrt{-\Lambda / 3}$.

In this model, we include the non-minimal E/M coupling required by $\mathcal{N}=2$ AdS SUGRA,

$$
\mathcal{L}_{\text {Non-Min }}=-\frac{i e}{m} \sqrt{-g} \bar{\psi}_{\mu} \mathcal{F}^{\mu \nu} \psi_{\nu}
$$

where

$$
\mathcal{F}^{\mu \nu}=-F_{+}^{\mu \nu} \equiv-\left(F^{\mu \nu}+i \gamma^{5} \widetilde{F}^{\mu \nu}\right) .
$$

Again we assume that the Rarita-Schwinger field is a probe moving in a combined gravitational and E/M background which satisfies the bosonic field equations of $\mathcal{N}=2$ AdS SUGRA

$$
D_{\mu} F^{\mu \nu}=0=G^{\mu \nu}+\kappa^{2} T^{\mu \nu}-\Lambda g^{\mu \nu} .
$$

Once again, we proceed by contradiction, i.e. $\xi^{2}=-1$, since the leading discontinuities still satisfy (11)-(14), but the crucial secondary constraint (15) is now modified as the Rarita-Schwinger field equation reads

$$
R^{\mu}=\gamma^{\mu \nu \rho} \mathcal{D}_{\nu} \psi_{\rho}-\frac{i e}{m} F_{+}^{\mu \nu} \psi_{\nu}=0
$$

To compute the secondary constraint $\mathcal{D} . R$ we first note that

$$
\mathcal{D} . F_{+} \cdot \psi=F_{+}^{\mu \nu} \mathcal{D}_{\mu} \psi_{\nu}-i m \gamma^{5} \gamma \cdot \tilde{F} . \psi
$$


Furthermore

$\gamma^{[\mu} R^{\nu]}=2 \mathcal{D}^{[\mu} \psi^{\nu]_{+}}-\gamma^{[\mu}\left((\not \mathcal{D}-m) \psi^{\nu]}-\left(\mathcal{D}^{\nu]}-m \gamma^{\nu]}\right) \gamma \cdot \psi\right)-\frac{i e}{m} \gamma^{[\mu} F_{+}^{\nu] \rho} \psi_{\rho}=0$,

where we have introduced the notation $X^{[\mu \nu]_{+}} \equiv X^{[\mu \nu]}+i \gamma^{5} \widetilde{X}^{\mu \nu}$ (note that $\left.X^{[\mu \nu]_{+}} Y_{\mu \nu}=(1 / 2) X^{[\mu \nu]_{+}} Y_{[\mu \nu]_{+}}\right)$. The terms in brackets are equal to (see equation (32) of [8]) $(i e / 2 m) \gamma_{\rho} \gamma^{\mu} F_{+}^{\rho \sigma} \psi_{\sigma}=(i e / m) F_{+}^{\mu \rho} \psi_{\rho}$ using the identity $\gamma \cdot F_{+} \cdot \psi=0$. Hence

$$
\mathcal{D}^{[\mu} \psi^{\nu]_{+}}=\frac{i e}{m} \gamma^{[\mu} F_{+}^{\nu] \rho} \psi_{\rho}
$$

and in turn

$$
\mathcal{D} \cdot F_{+} \cdot \psi=-i m \gamma^{5} \gamma \cdot \widetilde{F} \cdot \psi-\frac{i e}{m} \gamma \cdot T \cdot \psi
$$

Here we used $\left(F_{-} \cdot F_{+}\right)_{\mu \nu}=(F . F+\widetilde{F} \cdot \widetilde{F})_{\mu \nu}=-2 T_{\mu \nu}$ (with $F_{-}^{\mu \nu} \equiv F^{\mu \nu}-$ $\left.i \gamma^{5} \widetilde{F}^{\mu \nu}\right)$. The first term on the right hand side of (29) precisely cancels the troublesome second term in (15) and we find

$$
\mathcal{D} \cdot R=-\frac{3}{2} m^{2} \gamma \cdot\left(\psi+\frac{1}{3 m^{2}} G \cdot \psi+\frac{2 e^{2}}{3 m^{4}} T \cdot \psi\right) .
$$

For the discontinuity in the derivative of the secondary constraint we find (employing the background field equations (24))

$$
\xi^{\mu}\left[\partial_{\mu} \mathcal{D} \cdot R\right]_{\Sigma}=-\frac{3}{2} m^{2} \gamma \cdot\left[\xi\left(1+\frac{\Lambda}{3 m^{2}}\right)+T \cdot \xi\left(\frac{2 e^{2}}{3 m^{4}}-\frac{\kappa^{2}}{3 m^{2}}\right)\right] \xi . \Psi .
$$

Firstly note how the result of [11] is recovered: The SUGRA tuning of the $s=3 / 2$ mass

$$
m^{2}=\frac{2 e^{2}}{\kappa^{2}}
$$

maintains causality even when $\Lambda=0$ (and in fact for any cosmological constant). The point where in addition, $\Lambda=-3 m^{2}$ corresponds to unbroken $\mathcal{N}=2$ SUGRA whose $s=3 / 2$ field is genuinely massless with null propagation.

To study causality more generally, we require the determinant of the matrix multiplying $\xi . \Psi$ not to vanish. We now assume that the equality (32) does not hold. Again, by contradiction put $\xi_{\mu}=(1,0,0,0)$. Calling $\alpha \equiv 1+\Lambda / 3 m^{2}$ and $\mathcal{E} \equiv\left(\kappa^{2} / 2\right)\left(2 e^{2} / 3 m^{4}-\kappa^{2} / 3 m^{2}\right) \vec{E}^{2}$ and $\mathcal{B} \equiv\left(\kappa^{2} / 2\right)\left(2 e^{2} / 3 m^{4}-\kappa^{2} / 3 m^{2}\right) \vec{B}^{2}$ this determinant vanishes iff

$$
(\alpha-\mathcal{E})^{2}-2\left(\alpha-\mathcal{E}+2 \sin ^{2} \theta \mathcal{E}\right) \mathcal{B}+\mathcal{B}^{2}=0
$$


which implies the model is causal whenever the discriminant is negative, namely when

$$
\sin ^{2} \theta \mathcal{E}\left(\alpha-\cos ^{2} \theta \mathcal{E}\right)<0 .
$$

There are two cases. Firstly if

$$
m^{2}>\frac{2 e^{2}}{\kappa^{2}}
$$

then $\mathcal{E}<0$ and the model is causal so long as $\alpha>0$ which implies the additional restriction on the mass

$$
m^{2}>-\Lambda / 3
$$

Alternatively if

$$
m^{2}<\frac{2 e^{2}}{\kappa^{2}}
$$

then $\mathcal{E}>0$ and the model is causal only if $\alpha<0$, i.e. for masses

$$
m^{2}<-\Lambda / 3
$$

The corresponding $\left(m^{2}, \Lambda\right)$ phase diagram is that of Figure 3 .

\subsection{Non-minimal Couplings and Dynamical Rarita-Schwinger Fields}

We end this Section by considering the effect of general non-minimal couplings on the above results along with some comments on dynamical fermi fields.

In principle it is possible to add the most general non-minimal couplings along the lines of [8]; however as shown there, the most general magnetic moment couplings are already mapped out by non-minimal interactions of the form

$$
\mathcal{L}_{\text {Non-Min }}=-\frac{i e l}{m} \sqrt{-g} \bar{\psi} \cdot \mathcal{F} . \psi .
$$

Indeed, the parameter $l$ may be expressed in terms of the gyromagnetic ratio as [8]

$$
l=\frac{3 g-2}{4} .
$$

The supersymmetric case $l=1$ corresponds to $g=2$. 
The discontinuity in the derivative of the secondary constraint is modified to read

$$
\begin{aligned}
& \xi^{\mu}\left[\partial_{\mu} \mathcal{D} \cdot R\right]_{\Sigma}= \\
& \quad-\frac{3}{2} m^{2} \gamma \cdot\left[\left(1-\frac{\Lambda}{3 m^{2}}\right) \xi+\frac{2 e(1-l)}{3 m^{2}} \gamma^{5} \widetilde{F} \cdot \xi+\left(\frac{2 e^{2} l^{2}}{3 m^{4}}-\frac{\kappa^{2}}{3 m^{2}}\right) T \cdot \xi\right] \xi . \Psi .
\end{aligned}
$$

The analysis now proceeds in a fashion similar to that presented above. For simplicity we concentrate on the most physically relevant case, namely $\Lambda=0$ and $m<\sqrt{2} e / \kappa$. It easily verified that the relevant discriminant is a sum of squares (saturated at the supersymmetric point $l=1, m=\sqrt{2} e / \kappa$ )

$$
\left(\frac{2 e^{2} l^{2}}{m^{2}}-\kappa^{2}\right)^{2}+(2 e(1-l))^{2} \geq 0
$$

and can never be negative: non-minimal couplings cannot restore causality in the flat space, small mass regime.

Finally we consider the consequences of making the Rarita-Schwinger field dynamical. This requires the inclusion of the $s=3 / 2$ stress-energy tensor on the right hand side of Einstein's equations. In the case of SUGRA, the additional trilinear terms in the field equations from varying the four fermi terms required for local supersymmetry of the action will cancel the new contributions from the $s=3 / 2$ stress-energy tensor. For minimal coupling, however, one simply has to deal with these new terms. For example, if one views the classical value of spinor bilinears as an expectation value and assumes that $T_{3 / 2}^{00}>0$ then all additional contributions to the polynomial (19) are at least positive and will not weaken the resulting causality bounds.

\section{Spin 2}

We now analyze the first instance in which there is no underlying protection, in contrast to that provided by SUGRA for $s=3 / 2$ : while $s=2$ is a tensor theory, when charged it bears little resemblance to its one conceivable relative, Einstein gravity. Charged massive $s=2$ preserves the correct DoF only for gyromagnetic ratio $g=1 / 2$, but even this theory suffers from the usual causality difficulties. Furthermore, it has no good DoF coupling to general gravitational backgrounds, so there is no analog of the causality bounds found for $s=3 / 2$. 


\subsection{DoF Count}

The unique charged massive $s=2$ theory maintaining the correct $2 s+1=5$ (complex) DoF count in E/M backgrounds was discovered in [17]. To establish notation, we first write the free Lagrangian,

$$
\mathcal{L}=\frac{1}{2} \phi^{* \mu \nu} G_{\mu \nu}(\partial, \phi)-\frac{1}{2} m^{2}\left(\phi^{* \mu \nu} \phi_{\mu \nu}-\phi^{*} \phi\right)
$$

where $\phi \equiv \phi_{\mu}{ }^{\mu}$. The linearized Einstein tensor

$$
G_{\mu \nu}(\partial, \phi)=\square\left(\phi_{\mu \nu}-\eta_{\mu \nu} \phi\right)+\partial_{\mu} \partial_{\nu} \phi+\eta_{\mu \nu} \partial . \partial . \phi-2 \partial . \partial_{(\mu} \phi_{\nu)}, \quad \partial . \phi_{\mu} \equiv \partial^{\rho} \phi_{\rho \mu}
$$

satisfies the Bianchi identity

$$
\partial . G_{\mu}=0
$$

The free field equations are

$$
\mathcal{G}_{\mu \nu} \equiv\left(\square-m^{2}\right)\left(\phi_{\mu \nu}-\eta_{\mu \nu} \phi\right)+\partial_{\mu} \partial_{\nu} \phi+\eta_{\mu \nu} \partial . \partial . \phi-2 \partial . \partial_{(\mu} \phi_{\nu)}=0
$$

from which the usual on-shell conditions for massive $s=2$,

$$
\left(\square-m^{2}\right) \phi_{\mu \nu}=0=\partial . \phi_{\mu}=\phi
$$

follow upon using

$$
\partial . \partial . \mathcal{G}+\left(m^{2} / 2\right) \mathcal{G}_{\mu}{ }^{\mu}=\left(3 m^{4} / 2\right) \phi=0=\partial . \mathcal{G}_{\mu}=-m^{2}\left(\partial . \phi_{\mu}-\partial_{\mu} \phi\right) \text {. }
$$

The minimal coupling procedure $\partial_{\mu} \rightarrow D_{\mu}=\partial_{\mu}+i e A_{\mu}$ is ambiguous since possible reorderings of partial derivatives in the last term of (46) lead to a one parameter family of couplings (presciently labelled by the gyromagnetic ratio $g$ )

$$
\partial . \partial_{(\mu} \phi_{\nu)} \longrightarrow g D \cdot D_{(\mu} \phi_{\nu)}+(1-g) D_{(\mu} D \cdot \phi_{\nu)}=D_{(\mu} D \cdot \phi_{\nu)}+i e g F_{\rho(\mu} \phi_{\nu)}^{\rho} \text {. }
$$

[The particular choice $g=1$ corresponds to minimal coupling in a first order formalism in which

$$
-\frac{1}{2} G_{\mu \nu}=\partial_{\rho}{\Omega^{\rho}}_{\mu \nu}-\partial_{(\mu} \Omega_{\nu) \rho}^{\rho}-\frac{1}{2} \eta_{\mu \nu}\left(\partial_{\rho} \Omega_{\sigma}^{\rho \sigma}-\partial_{\rho} \Omega_{\sigma}^{\sigma \rho}\right)
$$

\footnotetext{
${ }^{3}$ See [15], 27] for a geometric formulation of free massless higher spin fields in terms of generalized Christoffel symbols.
} 
and the on-shell linearized Christoffel symbols are

$$
\left.\Omega_{\rho \mu \nu}=\partial_{(\mu} \phi_{\nu) \rho}-\frac{1}{2} \partial_{\rho} \phi_{\mu \nu} \cdot\right]
$$

To study DoF in an E/M background, we begin with the one parameter family of minimally coupled Lagrangians

$$
\begin{aligned}
\mathcal{L}= & -\frac{1}{2} D^{\rho} \phi^{* \mu \nu} D_{\rho} \phi_{\mu \nu}-\frac{1}{2} m^{2} \phi^{* \mu \nu} \phi_{\mu \nu}+\frac{1}{2} D^{\rho} \phi^{*} D_{\rho} \phi+\frac{1}{2} m^{2} \phi^{*} \phi \\
& -\frac{1}{2} D \cdot \phi^{* \mu} D_{\mu} \phi-\frac{1}{2} D^{\mu} \phi^{*} D \cdot \phi_{\mu}+D \cdot \phi^{* \mu} D \cdot \phi_{\mu}+i e g \phi^{* \rho \mu} F_{\mu \nu} \phi_{\rho}^{\nu} .
\end{aligned}
$$

As always with models involving constraints, it is necessary to first verify that interactions maintain the correct DoF. Here we must investigate the single and double divergences of the field equation

$$
\begin{aligned}
\mathcal{G}_{\mu \nu}= & \left(D^{2}-m^{2}\right)\left(\phi_{\mu \nu}-\eta_{\mu \nu} \phi\right)+D_{(\mu} D_{\nu)} \phi+\eta_{\mu \nu} D \cdot D \cdot \phi-2 D_{(\mu} D . \phi_{\nu)} \\
& -2 \operatorname{eig} F_{\rho(\mu} \phi_{\nu)}{ }^{\rho}=0 .
\end{aligned}
$$

We now examine candidate constraints arising from single and double divergences of the field equations, recast in terms of Lichnerowicz wave operators in the Appendix as

$$
\begin{aligned}
\mathcal{G}_{\mu \nu}= & \left(\Delta^{(2)}-m^{2}\right)\left(\phi_{\mu \nu}-\eta_{\mu \nu} \phi\right)+D_{(\mu} D_{\nu)} \phi+\eta_{\mu \nu} D \cdot D \cdot \phi-2 D_{(\mu} D \cdot \phi_{\nu)} \\
& +2 i e(2-g) F_{\rho(\mu} \phi_{\nu)}{ }^{\rho}=0 .
\end{aligned}
$$

To begin with, the single divergence

$$
\begin{aligned}
D \cdot \mathcal{G}_{\mu}= & -m^{2}\left(D \cdot \phi_{\mu}-D_{\mu} \phi\right) \\
& +i e\left\{\frac{1}{2} j_{\mu} \phi-(1-g) j \cdot \phi_{\mu}+g\left(\partial_{\rho} F_{\mu \nu}\right) \phi^{\nu \rho}\right. \\
& \left.+F_{\mu \nu}\left[(1+g) D \cdot \phi^{\nu}-\frac{3}{2} D^{\nu} \phi\right]+(2-g) F^{\nu \rho} D_{\rho} \phi_{\mu \nu}\right\}
\end{aligned}
$$

involves only first derivatives even in an E/M background with arbitrary choice of non-minimal couplings and is therefore a (Lagrangian) constraint. However, the double divergence

$$
D . D . \mathcal{G}+\frac{1}{2} m^{2} \mathcal{G}_{\mu}{ }^{\mu}=\frac{3}{2} m^{4} \phi-i e(1-2 g) F^{\mu \nu} D_{\mu} D . \phi_{\nu}
$$




$$
\begin{aligned}
+ & i e\{2 g j \cdot D \cdot \phi-j . D \phi \\
& \left.-(1-2 g)\left(\partial_{\mu} j_{\nu}\right) \phi^{\mu \nu}-2(1-g)\left(\partial_{\mu} F^{\rho}{ }_{\nu}\right) D_{\rho} \phi^{\mu \nu}\right\} \\
+ & e^{2}\left\{3 T_{\mu \nu} \phi^{\mu \nu}+(1+g) F_{\mu \rho} F^{\rho}{ }_{\nu} \phi^{\mu \nu}\right\}
\end{aligned}
$$

(where $T_{\mu \nu}=-F_{\mu}{ }^{\rho} F_{\rho \nu}+\frac{1}{4} \eta_{\mu \nu} F^{2}$ ) is crucial since it includes a term with an E/M field strength multiplying double time derivatives of fields

$$
-i e(1-2 g) F^{\mu \nu} D_{\mu} D . \phi_{\nu}
$$

This term implies a DoF breakdown since a constraint of the free model has become a propagating field equation. In particular, the leading time derivatives in (56) are -ie $(1-2 g) F^{0 i} \ddot{\phi}_{0 i}$ so that $F^{0 i} \phi_{0 i}$ becomes an additional propagating degree of freedom unless $g=1 / 2$. Therefore only the minimally coupled model with $g=1 / 2$ maintains the correct degree of freedom count. Curiously enough, this is the model that takes the average of the ambiguous terms $\mathrm{s}^{4}$ (49).

So far, we have seen that charged massive $s=2$ is uniquely described by the (local) Lagrangian

$$
\begin{aligned}
\mathcal{L}= & -\frac{1}{2} D^{\rho} \phi^{* \mu \nu} D_{\rho} \phi_{\mu \nu}-\frac{1}{2} m^{2} \phi^{* \mu \nu} \phi_{\mu \nu}+\frac{1}{2} D^{\rho} \phi^{*} D_{\rho} \phi+\frac{1}{2} m^{2} \phi^{*} \phi \\
& -\frac{1}{2} D \cdot \phi^{* \mu} D_{\mu} \phi-\frac{1}{2} D^{\mu} \phi^{*} D \cdot \phi_{\mu}+\frac{1}{2} D \cdot \phi^{* \mu} D \cdot \phi_{\mu}+\frac{1}{2} D^{\rho} \phi^{* \mu \sigma} D_{\sigma} \phi_{\mu \rho},
\end{aligned}
$$

with field equations

$$
\begin{aligned}
\mathcal{G}_{\mu \nu}= & \left(D^{2}-m^{2}\right)\left(\phi_{\mu \nu}-\eta_{\mu \nu} \phi\right)+D_{(\mu} D_{\nu)} \phi+\eta_{\mu \nu} D . D . \phi \\
& -D_{(\mu} D \cdot \phi_{\nu)}-D \cdot D_{(\mu} \phi_{\nu)}=0 .
\end{aligned}
$$

Correct DoF are ensured by the five constraint equations

$$
\begin{aligned}
D \cdot \mathcal{G}_{\mu}= & -m^{2}\left(D \cdot \phi_{\mu}-D_{\mu} \phi\right) \\
+ & \frac{i e}{2}\left\{3 F^{\nu \rho} D_{\rho} \phi_{\mu \nu}-j \cdot \phi_{\mu}\right. \\
& -3 F_{\mu \nu} D^{\nu} \phi+j_{\mu} \phi
\end{aligned}
$$

\footnotetext{
${ }^{4}$ The coupling $\mathcal{L}_{\mathrm{Non}-\mathrm{Min}} \propto \frac{i e}{2} \phi^{*} \rho \mu{ }_{\mu \nu} \phi^{\nu}{ }_{\rho}$ is the unique non-minimal term not involving derivatives on the matter fields. It is also not difficult to demonstrate that no coupling of the schematic form $\phi F D^{2} \phi$ involving two derivatives and an E/M field strength preserves the DoF count.
} 


$$
\begin{aligned}
& \left.+3 F_{\mu \nu} D \cdot \phi^{\nu}+\left(\partial_{\rho} F_{\mu \nu}\right) \phi^{\nu \rho}\right\}=0, \\
D . D . \mathcal{G}+\frac{1}{2} m^{2} \mathcal{G}_{\mu}{ }^{\mu}= & \frac{3}{2}\left(m^{4}-\frac{e^{2}}{2} F^{2}\right) \phi+\frac{3 e^{2}}{2} T_{\mu \nu} \phi^{\mu \nu} \\
& +i e(j . D \cdot \phi-j \cdot D \phi)+i e\left(\partial_{\mu} F_{\nu \rho}\right) D^{\rho} \phi^{\mu \nu}=0 .
\end{aligned}
$$

Of course a correct DoF count does not yet ensure consistency of the model since one still has to verify that the above constraints eliminate the unphysical DoF for all points in E/M field space. Equivalently, one can check the causality of the model, our next subject.

\subsection{Spin 2 Acausality}

Charged massive $s=2$ has long been known to propagate acausally [21, 22]. For completeness we provide a streamlined proof using the method of characteristics. Once again we examine the leading discontinuities of a shockwave, which are now second order and denoted as

$$
\left[\partial_{\mu} \partial_{\nu} \phi_{\rho \sigma}\right]_{\Sigma}=\xi_{\mu} \xi_{\nu} \Phi_{\rho \sigma}
$$

From the field equation and its trace we learn that

$$
\begin{aligned}
{\left[\mathcal{G}_{\mu \nu}-\frac{1}{2} \eta_{\mu \nu} \mathcal{G}_{\rho}{ }^{\rho}\right]_{\Sigma} } & =\xi^{2} \Phi_{\mu \nu}+\xi_{\mu} \xi_{\nu} \Phi-2 \xi_{(\mu} \xi . \Phi_{\nu)}=0 \\
{\left[\mathcal{G}_{\mu}{ }^{\mu}\right]_{\Sigma} } & =-2 \xi^{2} \Phi+2 \xi . \xi . \Phi=0
\end{aligned}
$$

$\left(\Phi \equiv \Phi_{\mu}{ }^{\mu}\right)$. We now study the system for acausal timelike normal vector $\xi^{2}=-1$. Note that since $\Phi_{\mu \nu} \neq 0$, we deduce that $V_{\mu} \equiv \xi . \Phi_{\mu} \neq 0$ (otherwise $\xi^{2}=0$ and the model would be causal). So we now impose

$$
\Phi_{\mu \nu}=-\xi_{\mu} \xi_{\nu} \xi . V-2 \xi_{(\mu} V_{\nu)}, \quad \Phi=-\xi . V
$$

and study further constraints. In particular, the single divergence constraint 60 ) gives

$$
\xi^{\mu}\left[\partial_{\mu} D \cdot \mathcal{G}_{\nu}\right]_{\Sigma}=m^{2}\left(V_{\nu}+\xi_{\nu} \xi . V\right)+\frac{3 i e}{2}\left(F_{\nu \rho} V^{\rho}-\xi^{\rho} F_{\rho \nu} \xi . V+\xi_{\nu} \xi . F . V\right)
$$

so that

$$
\Pi_{\nu \rho}\left[m^{2} \eta^{\rho \sigma}+\frac{3 i e}{2} F^{\rho \sigma}\right] \Pi_{\sigma \tau} V^{\tau}=0
$$


where the projector $\Pi_{\mu \nu} \equiv \eta_{\mu \nu}+\xi_{\mu} \xi_{\nu}$. It is sufficient to search for acausalities in constant background $\mathrm{E} / \mathrm{M}$ fields, so with this restriction the double divergence constraint gives

$$
\xi^{\mu} \xi^{\nu}\left[\partial_{\mu} \partial_{\nu}\left(D . D \cdot \mathcal{G}+\frac{1}{2} m^{2} \mathcal{G}_{\rho}{ }^{\rho}\right)\right]_{\Sigma}=-\frac{3}{2}\left(m^{4}-\frac{e^{2}}{2} F^{2}+e^{2} \xi . T . \xi\right) \xi . V-3 e^{2} \xi . T . V .
$$

Causality requires that the system of equations (67) and (68) have no non-zero solution for $V_{\mu}$. In general (67) implies the vanishing of the components of $V_{\mu}$ orthogonal to $\xi_{\mu}$ and (68) in turn removes the parallel components. However if, regarded as a matrix equation in the orthogonal subspace, equation (67) fails to remove the orthogonal components of $V_{\mu}$ the model will be acausal. The determinant in this subspace vanishes whenever

$$
\vec{B}^{2}=\left(\frac{2 m^{2}}{3 e}\right)^{2}
$$

This is the result of [21, 22]. Requiring that (68) be non-degenerate yields a different and weaker bound $\vec{B}^{2}=\left(2 m^{4}+\vec{E}^{2}\right) /\left(3 e^{2}\right)$. These differing bounds for the propagation of helicities zero and one are remiscent of the behaviour found for $s=2$ in cosmological backgrounds [3, 4 ].

There is no analog for $s=2$ of the $s=3 / 2$ improved causality behaviour in the presence of general gravitational backgrounds since the model cannot be coupled to gravity whilst maintaining the correct DoF [19, 20]. Essentially the most general double divergence constraint always becomes a propagating field equation even once non-minimal couplings are added. Clearly, the combination of dynamical Maxwell and Einstein fields is unlikely to yield an improvement of the situation since the possible double derivative terms in the charged $s=2$ double divergence constraint are not proportional to the E/M stress tensor. Furthermore, in [19, 20] consistent gravitational backgrounds have been found but they require that the traceless part of the Ricci tensor vanish, prohibiting a (trace-free) Maxwell stress tensor: Unlike the $s=3 / 2$ case, there is no underlying charged theory such as SUGRA to ensure consistency.

\subsection{Gyromagnetic Ratio and Tree Unitarity}

A very different requirement that has been imposed on higher spin theories is tree

unitarity [23]. The high energy behaviour of partial wave amplitudes is subject to 
unitarity bounds: $N$-point amplitudes should grow no faster than $E^{4-N}$ at high energies $E$. This requirement was used to analyze the uniqueness of spontaneously broken gauge field theories as a fundamental description of massive $s=1$ particles [28, 29]. [It is also important, although not sufficient, for renormalizability when higher loop diagrams are built from trees.] The precise mechanism is that terms proportional to inverse masses in propagators for spins $\geq 1$ generate a gauge transformation at the vertices. These terms are therefore cancelled by requiring vertices to satisfy gauge Ward identities. However, although this mechanism can be applied successfully to spins $<2$ [28, 29, 23] (for $s=3 / 2$ see also [8]), we now show that it in fact fails for $s=2$.

We begin by examining the massive $s=2$ propagator

$$
D_{\mu \nu, \rho \sigma}^{\mathrm{F}}(p)=\frac{-i}{p^{2}+m^{2}}\left[\Pi_{\mu \rho} \Pi_{\nu \sigma}-\frac{1}{3} \Pi_{\mu \nu} \Pi_{\rho \sigma}\right], \quad \Pi_{\mu \nu} \equiv \eta_{\mu \nu}+\frac{p_{\mu} p_{\nu}}{m^{2}}
$$

(suppressing obvious symmetrizations over $(\mu \nu)$ and $(\rho \sigma)$ on the right hand side). The terms in the numerator of the form

$$
\frac{p_{\mu} p_{\rho} \eta_{\nu \sigma}}{m^{2}}
$$

(plus permutations) must cancel in, for example, a tree level Compton scattering diagram since for generic kinematics they make unitarity-violating contributions $\sim E^{2}$. However, at a vertex, they amount to a gauge transformation,

$$
\delta \phi_{\mu \nu}=\partial_{(\mu} \xi_{\nu)}
$$

Hence a cancellation occurs if the leading E/M vertex is transverse with respect to this transformation. For spins 1 and 3/2, invariance (up to soft terms $\sim m$ or $\sim m^{2}$ ) with respect to a single derivative gauge invariance is sufficient for tree unitarity. However for $s \geq 2$, propagators involve increasing powers of $m^{-1}$. In particular for $s=2$, quartic terms

$$
\frac{p_{\mu} p_{\nu} p_{\rho} p_{\sigma}}{m^{4}}
$$

in the propagator numerator must cancel for tree unitarity. Therefore it is necessary to also require invariance of the vertex under a double derivative gauge transformation

$$
\delta \phi_{\mu \nu}=\partial_{(\mu} \partial_{\nu)} \xi
$$


For $s=2$, invariance of the leading E/M interaction couplings up to soft terms proportional to $\mathrm{m}^{2}$ when the on-shell conditions are imposed for any external lines would be sufficient to ensure tree unitarity.

Studying the E/M vertex with a single on-shell $s=2$ and photon line is equivalent to examining the terms in the field equation linear in $A_{\mu}$

$$
\left.\frac{1}{i e} \mathcal{G}_{\mu \nu}\right|_{A}=2 A . \partial \phi_{\mu \nu}+\eta_{\mu \nu}\left(\partial^{\rho} A^{\sigma}\right) \phi_{\rho \sigma}-2 \partial_{(\mu}\left(A . \phi_{\nu)}\right)-2 g F_{\rho(\mu} \phi_{\nu}{ }^{\rho} \text {. }
$$

Here we have imposed on-shell conditions

$$
\partial . \phi_{\nu}=0=\phi, \quad \partial . A=0
$$

and will also employ the usual

$$
\left(\square-m^{2}\right) \phi_{\mu \nu}=0=\square A_{\mu} .
$$

Furthermore we have reinstated general non-minimal couplings corresponding to the parameter $g \neq 1 / 2$ to see whether any value will help tree unitarity (even at the cost of an incorrect DoF count).

When the internal line attaches to terms (71) in the propagator, the single derivative gauge transformation (72) is induced so we must require a cancellation of the divergence of (75)

$$
\left.\frac{1}{i e} \partial \cdot \mathcal{G}_{\nu}\right|_{A}=(2-g) F^{\mu \rho} \partial_{\rho} \phi_{\mu \nu}-g\left(\partial_{\mu} F_{\rho \nu}\right) \phi^{\mu \rho}-m^{2} A . \phi_{\nu}
$$

The cancellation of the leading term at $g=2$ is that observed in [23], whilst the second term involving the gradient of the field strength can be cancelled by adding further (non-power renormalizable) E/M multipole couplings [23]. At this point it is tempting to require $g=2$ and declare the theory tree unitary since the final term proportional to $m^{2}$ is apparently soft.

However, we must still cancel the most dangerous terms (73) whose induced double derivative gauge transformation (74) necessitates a vanishing double divergence of (75). Even for $g=2$ and ignoring gradients of $F_{\mu \nu}$ we find

$$
\frac{1}{i e} \partial .\left.\partial \cdot \mathcal{G}\right|_{A}=-m^{2}\left(\partial^{\mu} A^{\nu}\right) \phi_{\mu \nu} .
$$

This term is hard in the photon momentum $k_{\mu}$ and violates tree unitarity. Clearly this is a disease generic to all higher spins with increasing powers of $m^{-1}$ appearing in propagators. 
Finally to verify that the parameter $g$ is indeed the gyromagnetic ratio, we look at the photon emission amplitude computed by taking all lines in the E/M vertex on-shell. In terms of on-shell $s=2$ polarizations $u_{\mu \nu}$ and $u_{\mu \nu}^{*}$ one finds

$$
T_{f i}=i e g F_{\mu \nu} u^{* \rho \mu} u_{\rho}^{\nu}
$$

which is consistent with low energy theorems (see [8] for details)

$$
T_{f i}=(i \mu / 2 s) F_{\mu \nu} \mathcal{M}^{\mu \nu}
$$

where the Lorentz generators in our covariant representation are

$$
\mathcal{M}^{\mu \nu} \equiv 2 m s u^{* \rho[\mu} u_{\rho}^{\nu]}
$$

and the magnetic moment of a spin $s$ particle is

$$
\mu=(\text { egs } / 2 m)
$$

Since the above result is deduced from an overall normalization only, we also studied the soft photon limit of the $s=2$ Compton scattering amplitude and found precise agreement with low energy theorems [30, 31, 32] for the identification of the gyromagnetic ratio quoted above. We also confirmed the above tree unitarity failure independently by evaluating an explicit Compton amplitude.

To summarize, the DoF-preserving model of [17] yields $g=1 / 2$ and no choice of gyromagnetic ratio, DoF-preserving or not, yields a tree unitary model. For effective phenomenological applications, tree unitarity only signals the scale at which the effective description breaks down. However, only the $g=1 / 2$ model preserves DoF and thereby offers a reliable effective perturbative description. In any case, even this theory lacks full consistency due to its causality difficulties. The phenomenogical usefulness of the pure $s=2$ model coupled to electromagnetism therefore seems rather limited.

\section{Conclusions}

We have reexamined $\mathrm{E} / \mathrm{M}$ and (when possible) gravitational interactions of massive higher spin fields. There are two possible obstructions to consistency. The first is a violation of the constraints leading to unphysical ghostlike propagating degrees of freedom. For $s=3 / 2$ this difficulty is easily avoided, but for $s=2$ it already implies a unique $g=1 / 2$ magnetic moment coupling and rules 
out consistent interactions with combined Maxwell-Einstein backgrounds. The second problem, relevant only when the first is absent, is a breakdown of causality/unitarity.

For $s=3 / 2$ we have presented new causality bounds in Maxwell-Einstein backgrounds. Allowing the electromagnetic background to interact with an Einstein one does improve the causality properties of the $s=3 / 2$ system. Indeed, one no longer needs to place unphysical bounds on the E/M field strength, but rather only on the parameters $\left(m^{2}, e, \kappa, \Lambda\right)$ of the theory . The resulting $\left(m^{2}, \Lambda\right)$ causality/unitarity phase diagrams (Figures 2, 3) are generalizations of those found for

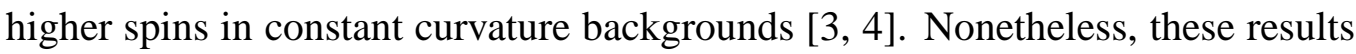
provide no solace in the search for a consistent effective theory of massive $s=3 / 2$ with realistic values of the mass $m^{2}$ and cosmological constant $\Lambda$. We also noted that the improved properties of massive $s=3 / 2$ are probably simply indications that it is a softly enough broken version of an underlying consistent theory: $\mathcal{N}=2$ cosmological SUGRA.

For $s=2$ there is much less to be done: Maxwell-Einstein causality analysis is not even applicable as the DoF problem strikes first! The only E/M model remaining is the gyromagnetic ratio $g=1 / 2$ one of [17] and for completeness we have included a derivation of its causality failure using the method of characteristics. There have been suggestions that $g=2$ ought to yield preferable behaviour. Certainly, this is plausible for an ultimately consistent description of relativistic charged higher spins because: (i) Optical and low energy theorems imply $g=2$ [32]. (ii) Massive higher spin string states couple to E/M backgrounds with $g=2$ [23]. (iii) Fundamental $s \leq 1$ particle excitations observed to date couple with $g=2$. We are not proposing the pure charged $s=2$ system as a counterexample to the $g=2$ folklore, but rather reiterate that it is simply not a consistent theory. Our finding that no gyromagnetic ratio yields tree unitary amplitudes is hardly surprising in this light. Instead, a whole Regge trajectory, as in string theory, is likely to be necessary for tree unitarity to hold [33]. In that context, it is reasonable to speculate that $g=2$ is germane to all spins [23].

An obvious open problem is to show that no finite tower of massive local higher spins can couple consistently to electromagnetism. A simpler version of this problem would be to demonstrate explicitly that spins $5 / 2$ or 3 cannot be coupled to E/M backgrounds in a DoF preserving way. Preliminary investigations indicate that this is probably the case; there is simply no non-minimal coupling available that can restore the consistency of the ever increasing set of constraints required for higher spins. For example, massive $s=3$ depends on constraints built from single, double and triple divergences of the field equations and the 
latter two are liable to become a propagating field equations for unphysical DoF when interactions are added. Clearly this difficulty is only compounded when $s$ increases.

\section{Acknowledgements}

It is a pleasure to thank Bernard de Wit and especially Massimo Porrati for discussions in the early stages. This work was supported by the National Science Foundation under grant PHY99-73935.

\section{Appendix: E/M Lichnerowicz Wave Operators}

It is well known that in constant curvature backgrounds, the algebra of covariant derivatives $D_{\mu}$ and the Laplacian $D^{2}$ is vastly simplified by introducing the wave operators of Lichnerowicz [34]. The same construction can be generalized to the case of electromagnetic backgrounds: Introduce the operators $\Delta^{(n)}$ acting on symmetric $n$-index tensors $\phi_{\mu_{1} \ldots \mu_{n}}$ as

$$
\Delta^{(n)} \phi_{\mu_{1} \ldots \mu_{n}} \equiv D^{2} \phi_{\mu_{1} \ldots \mu_{n}}+2 \operatorname{ien} F_{\left(\mu_{1}\right.}{ }^{\sigma} \phi_{\left.\mu_{2} \ldots \mu_{n}\right) \sigma}
$$

It is not difficult to show that the following properties hold

$$
\begin{aligned}
\eta^{\mu_{1} \mu_{2}} \Delta^{(n)} \phi_{\mu_{1} \ldots \mu_{n}} & =\Delta^{(n-2)} \phi_{\mu \mu_{3} \ldots \mu_{n}}^{\mu}, \quad(n \geq 2) \\
\Delta^{(n)} \eta_{\left(\mu_{1} \mu_{2}\right.} \phi_{\left.\mu_{3} \ldots \mu_{n}\right)} & =\eta_{\left(\mu_{1} \mu_{2}\right.} \Delta^{(n-2)} \phi_{\left.\mu_{3} \ldots \mu_{n}\right)}, \\
D^{\mu_{1}} \Delta^{(n)} \phi_{\mu_{1} \ldots \mu_{n}} & =\Delta^{(n-1)} D \cdot \phi_{\mu_{2} \ldots \mu_{n}} \\
& +i e j \cdot \phi_{\mu_{2} \ldots \mu_{n}}-2 i e(n-1)\left(\partial_{\rho} F_{\sigma\left(\mu_{2}\right.}\right) \phi_{\left.\mu_{3} \ldots \mu_{n}\right)}{ }^{\rho \sigma}, \quad(n \geq 1) \\
\Delta^{(n)} D_{\left(\mu_{1}\right.} \phi_{\left.\mu_{2} \ldots \mu_{n}\right)} & =D_{\left(\mu_{1}\right.} \Delta^{(n-1)} \phi_{\left.\mu_{2} \ldots \mu_{n}\right)}+i e j_{\left(\mu_{1}\right.} \phi_{\left.\mu_{2} \ldots \mu_{n}\right)},
\end{aligned}
$$

where $j_{\mu} \equiv \partial^{\rho} F_{\rho \mu}$ is the E/M current. These identities simplify further for constant E/M field strengths.

\section{References}

[1] S. Deser and A. Waldron, Phys. Lett. B513 (2001) 137, hep-th/0105181, 
[2] S. Deser and A. Waldron, Phys. Lett. B508 (2001) 347, hep-th/0103255,

[3] S. Deser and A. Waldron, Nucl. Phys. B607 (2001) 577, hep-th/0103198,

[4] S. Deser and A. Waldron, Phys. Rev. Lett. 87 (2001) 031601, hepth/0102166,

[5] S. Deser and A. Waldron, Phys. Lett. B501 (2001) 134, hep-th/0012014,

[6] K. Johnson and E. Sudarshan, Annals Phys. 13 (1961) 126.

[7] G. Velo and D. Zwanziger, Phys. Rev. 186 (1969) 1337,

[8] S. Deser, V. Pascalutsa and A. Waldron, Phys. Rev. D62 (2000) 105031, hep-th/0003011,

[9] P.K. Townsend, Phys. Rev. D15 (1977) 2802,

[10] D.Z. Freedman and A. Das, Nucl. Phys. B120 (1977) 221,

[11] S. Deser and B. Zumino, Phys. Rev. Lett. 38 (1977) 1433,

[12] C. Fronsdal, Phys. Rev. D18 (1978) 3624,

[13] J. Fang and C. Fronsdal, Phys. Rev. D18 (1978) 3630,

[14] T. Curtright, Phys. Lett. B85 (1979) 219,

[15] B. de Wit and D.Z. Freedman, Phys. Rev. D21 (1980) 358,

[16] L.P.S. Singh and C.R. Hagen, Phys. Rev. D9 (1974) 898,

[17] P. Federbush, Nuov. Cim. 19 (1961) 572.

[18] C. Aragone and S. Deser, Nuovo Cim. B57 (1980) 33,

[19] I.L. Buchbinder et al., (1999), hep-th/9910188,

[20] I.L. Buchbinder, D.M. Gitman and V.D. Pershin, (2000), hep-th/0006144,

[21] M. Kobayashi and A. Shamaly, Phys. Rev. D17 (1978) 2179,

[22] M. Kobayashi and A. Shamaly, Prog. Theor. Phys. 61 (1979) 656,

[23] S. Ferrara, M. Porrati and V.L. Telegdi, Phys. Rev. D46 (1992) 3529, 
[24] A. Shamaly and A.Z. Capri, Ann. Phys. 74 (1972) 503.

[25] J. Madore and W. Tait, Commun. Math. Phys. 30 (1973) 201.

[26] J. Madore, Phys. Lett. B55 (1975) 213.

[27] T. Damour and S. Deser, Ann. Inst. H. Poincaré 47 (1987) 277,

[28] C.H.L. Smith, Phys. Lett. B46 (1973) 233,

[29] J.M. Cornwall, D.N. Levin and G. Tiktopoulos, Phys. Rev. D10 (1974) 1145 ,

[30] F.E. Low, Phys. Rev. 96 (1954) 1428,

[31] M. Gell-Mann and M.L. Goldberger, Phys. Rev. 96 (1954) 1433,

[32] S. Weinberg, Lectures on Elementary Particles and Quantum Field Theory, Volume 1, 1970.

[33] A. Cucchieri, M. Porrati and S. Deser, Phys. Rev. D51 (1995) 4543, hepth/9408073,

[34] A. Lichnerowicz, Pub. Math. IHES 10 (1961) 293. 